\title{
Single-Unit Activity in Piriform Cortex during Slow-Wave State Is Shaped by Recent Odor Experience
}

\author{
Donald A. Wilson ${ }^{1,2,3}$ \\ ${ }^{1}$ Emotional Brain Institute, Nathan Kline Institute for Psychiatric Research, Orangeburg, New York 10962, ${ }^{2}$ Child and Adolescent Psychiatry, New York

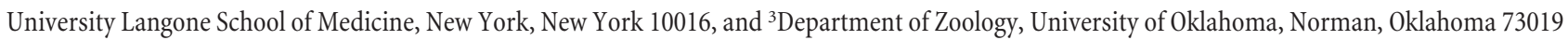

Memory and its underlying neural plasticity play important roles in sensory discrimination and cortical pattern recognition in olfaction. Given the reported function of slow-wave sleep states in neocortical and hippocampal memory consolidation, we hypothesized that activity during slow-wave states within the piriform cortex may be shaped by recent olfactory experience. Rats were anesthetized with urethane and allowed to spontaneously shift between slow-wave and fast-wave states as recorded in local field potentials within the anterior piriform cortex. Single-unit activity of piriform cortical layer II/III neurons was recorded simultaneously. The results suggest that piriform cortical activity during slow-wave states is shaped by recent (several minutes) odor experience. The temporal structure of single-unit activity during slow waves was modified if the animal had been stimulated with an odor within the receptive field of that cell. If no odor had been delivered, the activity of the cell during slow-wave activity was stable across the two periods. The results demonstrate that piriform cortical activity during slow-wave state is shaped by recent odor experience, which could contribute to odor memory consolidation.

\section{Introduction}

Spatial memories are replayed within the hippocampal formation during sleep (Pavlides and Winson, 1989; Skaggs and McNaughton, 1996; Louie and Wilson, 2001). Neurons encoding sequences of recently experienced spatial patterns are reactivated during slow-wave sleep (Lee and Wilson, 2002). This replay is hypothesized to facilitate consolidation of spatial memory, as well as help link hippocampal activity to neocortical activity (Buzsáki, 1996; Maquet, 2001; Lee and Wilson, 2002; Steriade and Timofeev, 2003; Eschenko et al., 2008; Cai et al., 2009; Wierzynski et al., 2009).

Like the hippocampal formation, the piriform cortex is a three-layered cortical structure with highly distributed, sparse encoding of input patterns (Haberly, 2001; Rennaker et al., 2007; Poo and Isaacson, 2009). The inputs to the piriform cortex are spatiotemporal patterns generated by the olfactory bulb in response to odor (Wachowiak and Cohen, 2001; Lin et al., 2006; Johnson and Leon, 2007). Through a combination of afferent convergence, a highly auto-associative intrinsic fiber system, and Hebbian synaptic plasticity, the anterior piriform cortex (aPCX) is hypothesized to develop experience-dependent memories of previously experienced input patterns to facilitate odor discrimination and recognition (Barkai et al., 1994; Wilson, 2001; Linster et al., 2009).

Olfactory experience induces a variety of changes throughout the olfactory pathway, including changes in local [e.g., within

\footnotetext{
Received Nov. 13, 2009; revised Dec. 8, 2009; accepted Dec. 11, 2009.

This work was supported by National Institutes of Health Grant R01 DC003906 (D.A.W.).

Correspondence should be addressed to Donald A. Wilson, Nathan Kline Institute for Psychiatric Research, 140 Old Orangeburg Road, Orangeburg, NY 10962. E-mail: donald.wilson@nyumc.org.

DOI:10.1523/JNEUROSCI.5636-09.2010

Copyright $\odot 2010$ the authors $\quad 0270-6474 / 10 / 301760-06 \$ 15.00 / 0$
}

cortical areas (Wilson et al., 1987; Grajski and Freeman, 1989; Ravel et al., 2003; Quinlan et al., 2004)] and global [e.g., between cortical areas (Martin et al., 2006, 2007; Cohen et al., 2008)] circuit connectivity. One of the consequences of these changes is olfactory perceptual learning, which is an enhancement in perceptual acuity for the learned odor and enhanced discrimination of that odor from similar odors (Stevenson, 2001; Fletcher and Wilson, 2002; Wilson, 2003; Wilson and Stevenson, 2003; Li et al., 2008). Olfactory perceptual learning and modulation of odor acuity are associated with changes in both the olfactory bulb (Fletcher and Wilson, 2003; Mandairon et al., 2006; Doucette et al., 2007; Doucette and Restrepo, 2008; Chaudhury et al., 2009) and aPCX (Wilson, 2003; Li et al., 2008). In aPCX, both singleunits (Wilson, 2000, 2003), and single-unit ensembles (Kadohisa and Wilson, 2006) show enhanced decorrelation of familiar odors even after passive exposure to those odors (Wilson, 2003). In fact, under urethane anesthesia, brief passive exposure to novel odors is sufficient to improve piriform cortical single-unit discrimination (Wilson, 2003). Disruption of normal aPCX association fiber synaptic transmission and plasticity, for example by disrupting cholinergic input (Barkai and Hasselmo, 1994; Patil et al., 1998), impairs olfactory perceptual learning at both the cortical (Wilson, 2001; Linster et al., 2009) and behavioral (Fletcher and Wilson, 2002) level.

We hypothesized that activity during slow-wave states within the aPCX may be shaped by recent olfactory experience. Specifically, we took advantage of the fact that urethane-anesthetized rats undergo spontaneous shifts between fast-wave and slowwave states (Murakami et al., 2005). Both single-unit and local field potential (LFP) activity were monitored within the aPCX during slow-wave states before and after a fast-wave state during which repeated odor stimulation was delivered. The results sug- 
A
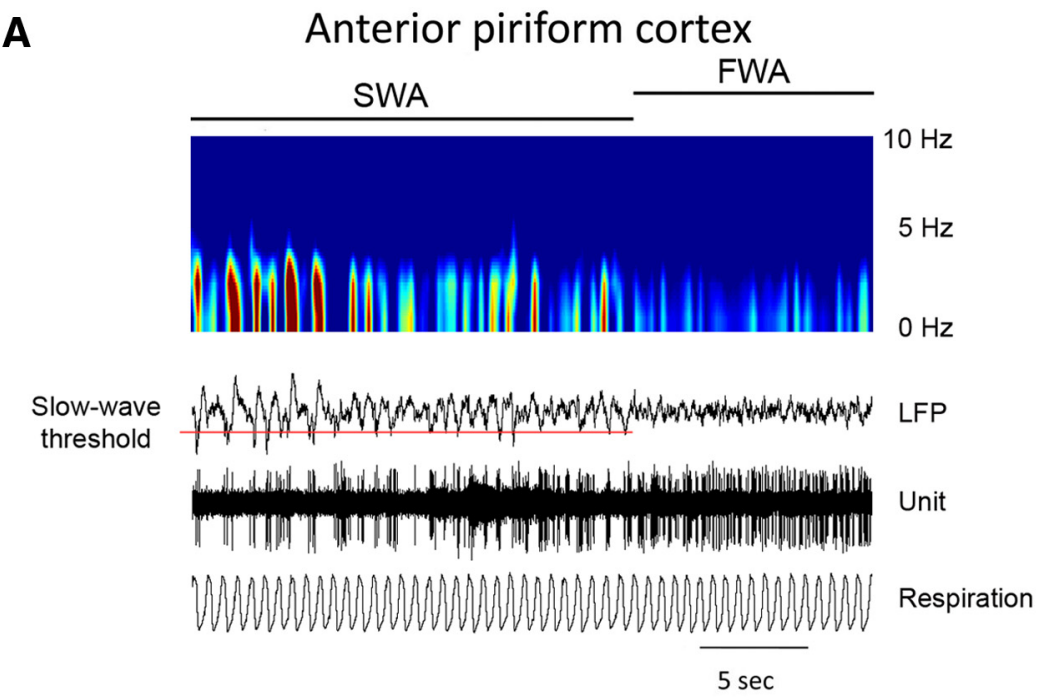

B
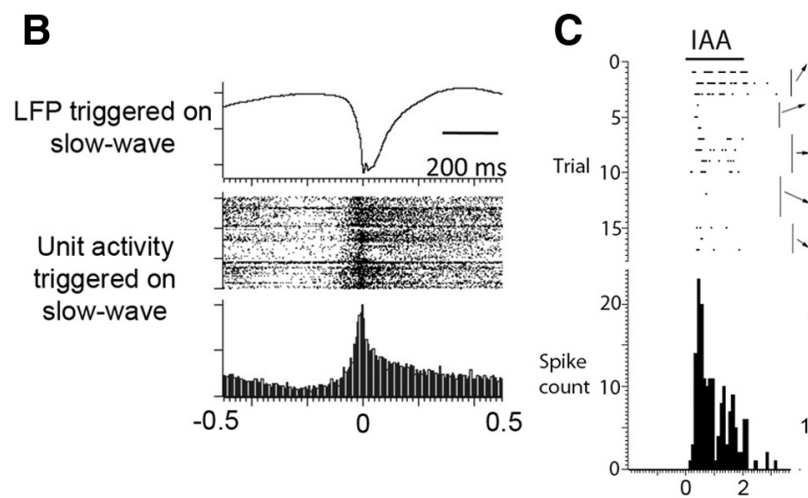

Spont. LFP

Whather FWA

were approved by the institutional animal care and use committees at both the Nathan Kline Institute and the New York University Medical School. Single units (filtered at $300 \mathrm{~Hz}$ to $3 \mathrm{kHz}$ ) and LFPs (filtered at $0.1-300 \mathrm{~Hz}$ ) were recorded simultaneously with a single tungsten microelectrode (1-5 M $\Omega$ ). Signals were digitized at $10 \mathrm{kHz}$ with a CED micro1401 and analyzed with Spike2 software (both from Cambridge Electronic Design). Electrode placement in the aPCX was guided by recording potentials evoked by electrical stimulation of the lateral olfactory tract and confirmed histologically at the end of the experiment. Respiration was monitored with a piezoelectric sensor strapped to the chest. This allowed both recording of the respiration and acted as a trigger for controlling odorant stimulation onset. Anesthetic plane was such that no odorantevoked respiratory responses were detected. Odorants were delivered in $2 \mathrm{~s}$ pulses initiated at the transition from inhalation to exhalation. Monomolecular odorants were delivered at $100 \mathrm{ppm}$ based on vapor pressure and diluted in mineral oil. At least 30-60 s interstimulus intervals were used to reduce cortical adaptation. Odorants included the following: isoamyl acetate, ethyl valerate, 5-methyl-2-hexanone, 1-pentanol, heptanal, 2-heptanone, and 1,7-octadiene.

The work was divided into two experiments. First, in four animals, odor stimuli were delivered during both slow-wave (SWA) and fast-wave (FWA) activity. State changes were readily apparent by eye in the LFPs and were confirmed quantitatively by fast Fourier transform analyses. SWA was dominated by strong delta frequency (1-4 $\mathrm{Hz}$ ) power and large slow waves that occurred at 1-2 Hz. FWA had reduced delta, higher theta $(4-12 \mathrm{~Hz})$, and beta $(15-30 \mathrm{~Hz})$ frequency activity and no slow waves. This experiment was a replication of the study of Murakami et al. (2005) to confirm differential cortical responsiveness to afferent input during SWA and FWA.

The main experiment involved odor stimulation only during FWA to allow comparisons between spontaneous SWA before and after FWA odor stimulation. This design was meant to mimic the analyses performed in the hippocampal formation wherein spontaneous activity is monitored during SWA before and after maze learning, which occurs when the animal is awake (Pavlides and Winson, 1989; Skaggs and McNaughton, 1996). Once a single unit or multiple single units were isolated, the animal was allowed to transition into SWA. To be included, an SWA period had to last long enough to have at least 90 slow waves. Once the animal had transitioned to FWA, cells were randomly

assigned to either receive odor stimulation or no

Figure 1. A, LFP recordings in aPCX revealed spontaneous shifts between SWA and FWA as reported previously (Murakami et al., 2005). The top shows a pseudocolor plot of the LFP fast Fourier transform analysis filtered to emphasize the large delta frequency $(1-4 \mathrm{~Hz})$ activity during SWA. B, During SWA, single units fired in-phase with the slow waves as shown in although different cells had peak activity at different phases of the wave. C, Single-unit odor responses were reduced during SW compared with FWA. The raster plot and LFPs show that, as an animal cycles through FWA and SWA, odor response magnitude similarly cycles. There was a gradual habituation across repeated FWA states. For direct comparison, responses to the first six stimuli in each state are shown side by side. $\boldsymbol{D}$, Single-unit and LFP activity were decoupled from respiration during SWA compared with FWA. Histograms show respiratory events as a function of single-unit spiking (at arrowheads). LFPs are mean waveforms triggered on single-unit spikes (at arrowheads). Overdrawn waveform of single unit used for analysis in $\boldsymbol{D}$ in shown at bottom.

gest that slow-wave state activity within the aPCX reflects recent odor experience.

\section{Materials and Methods}

Single-unit recordings were made from layer II/III neurons in aPCX of urethane $(1.5 \mathrm{~g} / \mathrm{kg})$ male Long-Evans hooded rats as described previously (Wilson, 1998). Rats were obtained from Charles River Laboratories. Recordings were made during the light phase of the $12 \mathrm{~h}$ light/dark cycle. Animals had ad libitum access to food and water and were cared for according to National Institutes of Health guidelines. All procedures odor stimulation. To be included in the odor-stimulation dataset, units had
to show a significant and reliable response to at least one odor. If a cell was tested but shown to be nonresponsive to any odor (no more than five total test stimuli, or tested at the end of a control session), it and the animal were excluded from the analysis. In some cases, multiple single units were recorded simultaneously, and data were acquired from all. However, in no case were multiple cells within the same animal tested sequentially, because previous odor experiences may have affected later tests.

Single-unit activity during SWA was assessed with peri-slow-wave time histograms (PSWTHs). Slow waves were extracted from continuous 
Table 1. Characteristics of slow waves before and after FWA in the two groups

\begin{tabular}{|c|c|c|c|c|c|c|}
\hline Group & Number of units & $\begin{array}{l}\text { Number of slow waves } \\
\text { sampled before }\end{array}$ & $\begin{array}{l}\text { Number of slow waves } \\
\text { sampled after }\end{array}$ & $\begin{array}{l}\text { Slow-wave rate } \\
(\mathrm{Hz}) \text { before }\end{array}$ & $\begin{array}{l}\text { Slow-wave rate } \\
(\mathrm{Hz}) \text { after }\end{array}$ & $\begin{array}{l}\text { FWA duration between } \\
\text { before and after (min) }\end{array}$ \\
\hline Control & 22 & $266 \pm 36$ & $267 \pm 37$ & $0.41 \pm 0.04$ & $0.39 \pm 0.03$ & $35.8 \pm 3.8$ \\
\hline Odor & 22 & $283 \pm 25$ & $284 \pm 25$ & $0.61 \pm 0.16$ & $0.43 \pm 0.03$ & $38.2 \pm 5.5$ \\
\hline
\end{tabular}

The duration of sampled SWA was maintained constant across time and groups to allow comparisons under constant conditions. None of the differences between control and odor recordings are significant ( $t$ test).

LFPs that were low-pass filtered at $50 \mathrm{~Hz}$. The time stamps of slow-wave peaks (identified by thresholding in each individual animal) were used as triggers to build PSWTHs from ongoing single-unit activity (Fig. $1 A, B$ ). PSWTHs ( $5 \mathrm{~ms}$ bin width) were built for each cell during two SWA periods. Two measures were calculated for comparison of activity during these two periods. First, total activity within \pm 100 $\mathrm{ms}$ of the slow-wave peak was determined. Second, the peak of single-unit activity during the PSWTH $( \pm 100 \mathrm{~ms}$ of slow-wave peak) was compared between the two SWA periods to determine whether single-unit activity during the slow wave shifted between time periods. The time bin containing the highest spike count was considered the peak. If two to three nearby time bins were of equal counts, the average time between them was used to calculate the peak shift. If four or more peaks were equal or if the peaks were widely scattered, that cell was eliminated from this calculation. Five single units in the control group and three single units in the odorstimulated group were dropped for this reason from this specific calculation.

Slow-wave morphology was determined by calculating an average LFP triggered on the slow-wave peak $( \pm 500 \mathrm{~ms})$. Individual animal mean waveforms were then averaged within sample periods and within experimental groups for statistical comparisons (ANOVA).

\section{Results}

In four rats separate from those used in the primary experiment here, we confirmed that aPCX sensory-evoked activity is markedly different during FWA and SWA. As described previously (Murakami et al., 2005) and as shown in Figure $1 A$, LFPs recorded in the aPCX spontaneously shifted between periods of FWA and SWA, with transitions in either direction often occurring rapidly over 1-2 s. As shown in Figure $1 B$, during SWA, single units recorded in aPCX layers II/III fired in-phase with the slow waves, most commonly with firing peaking near its nadir. Furthermore, during SWA, single units showed reduced responsiveness to odors (Fig. 1C) and reduced entrainment to the respiratory cycle (Fig. 1D) compared with during FWA. Single-unit response magnitude (numbers of spikes/stimulus above baseline rate) to odors presented during SWA was reduced to $31.3 \pm 13.8 \%$ of response magnitude to the same odors presented during FWA (paired $t$ test, $t_{(7)}=3.83, p<$ 0.01 ). The reduction in responsiveness to afferent input has been shown to be attributable to changes intrinsic to the aPCX, because responsivity of cortical afferent mitral cells to odors is not reduced during SWA (Murakami et al., 2005). For data presented below, odors were not presented during SWA.

The main question to be examined here was whether spontaneous activity during SWA was modified by recent FWA odor experi- ence, similar to that described for the hippocampus. Data from 44 single units ( 22 odor-exposed and 22 control) from 29 rats are described. Slow-wave-associated spontaneous single-unit activity was monitored during two SWA periods. In odor-exposed cells, during the FWA between these two SWA periods, odor stimuli to which the unit responded were repeatedly delivered. The mean delay between the last odor stimulus and the onset of SWA measurements was $360 \mathrm{~s}$, with a range of $1-30 \mathrm{~min}$. In control cells, no stimulation was delivered during the intervening FWA. Periods of SWA were chosen to equate the number of slow waves that occurred during the two periods within a cell and between the two groups (Table 1). As shown in Table 1, there was no significant difference between slowwave rate or duration of the intervening FWA period between groups ( $t$ tests).

Single-unit activity associated with slow waves was significantly modified by previous odor experience compared with control cells that were not odor stimulated. Mean slow-wave as- 


\section{Control}

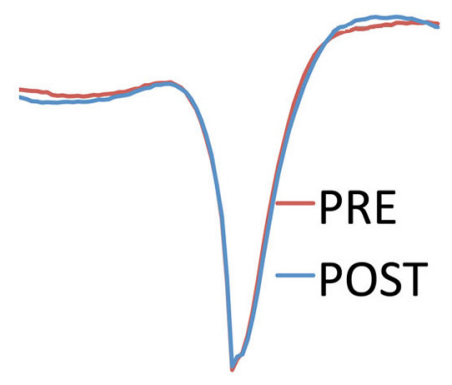

Odor-exposure

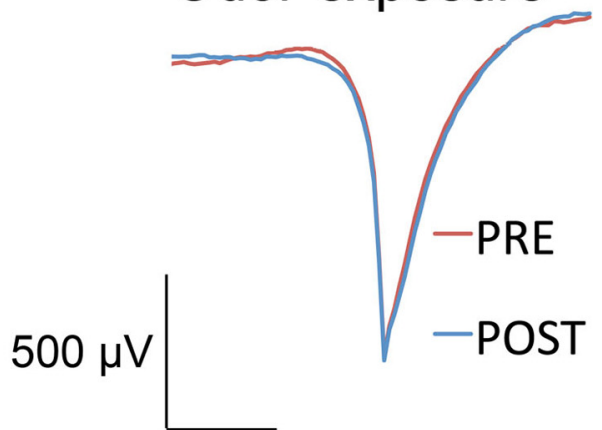

$250 \mathrm{~ms}$

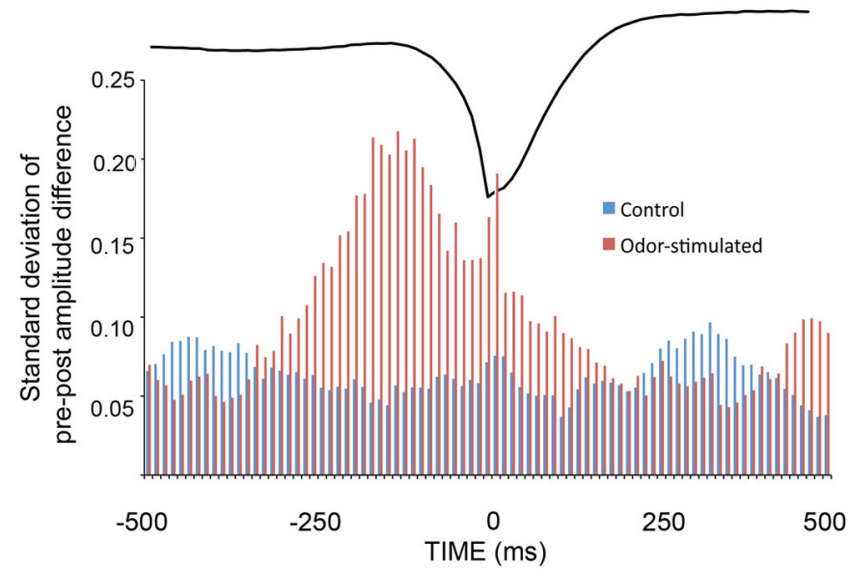

Figure 3. Top, There was no significant change in mean slow-wave morphology in either control or odor-stimulated animals between pre- and post-odor time points. Bottom, However, variability in the leading edge of the slow wave was significantly enhanced by previous odor stimulation. This variability in slow-wave morphology was associated with a significant shift of peak single-unit activity during the slow wave after odor stimulation compared with single units in controls.

cantly greater shift in slow-waveassociated peak firing during the two sampling periods (odor-exposed mean shift, $50.4 \pm 9 \mathrm{~ms}$; control shift, $20.9 \pm 5$ ms; $t$ test, $\left.t_{(33)}=2.43, p=0.02\right)$. The direction of the shift in peak slow-waveassociated activity was equally balanced between earlier or later times. That is, some cells fired earlier during the slow wave after odor exposure and some fired later $(50 \%$ earlier and $50 \%$ later in the odor-exposed cells). In a small set of five cells, odors were delivered during FWA that were outside of the receptive field of the cells and thus produced no odorevoked change in firing. Although a small sample, the activity of these cells during the subsequent SWA period was similar to that of the unstimulated controls, with a small mean shift of $27 \mathrm{~ms}$ (compared with $21 \mathrm{~ms}$ for the unstimulated controls and $50 \mathrm{~ms}$ for the stimulated cells).

Given that the slow waves recorded in the LFP reflect activity of a large population of neurons and a subset of those neurons change their activity relative to the population based on recent odor experience (Fig. 2), there may be a concomitant change in the slow waves themselves. As shown in Figure 3, there was no change in mean peak amplitude or overall morphology of the slow waves between the two SWA sampling periods. However, an analysis of the difference in pre- versus post-waveforms (mean post-waveform

sociated activity (PSWTH) across all single units within each group is plotted in Figure 2. In control cells, mean slow-waveassociated activity did not significantly change between the two periods of SWA (Fig. 2 B). However, in odor-stimulated animals, mean slow-wave-associated activity was significantly reduced (paired $t$ tests, $p<0.05$ ) during SWA after odor stimulation compared with SWA before odor stimulation (Fig. 2C).

The observed decrease in mean slow-wave-associated activity in the odor-exposed cells could be attributable to either a decrease in individual unit activity after odor exposure or a shift in the temporal structure of individual unit activity during the slow wave, with some cells firing earlier and some later than under baseline conditions. The change in slow-wave-associated activity was not attributable to a change in activity rate. A repeated-measures ANOVA of total singleunit spiking during the $200 \mathrm{~ms}$ period surrounding ( $\pm 100 \mathrm{~ms})$ a slow-wave peak showed no significant effect of either group (odorexposed vs control, $F_{(1,38)}=0.30$, NS) or time (before vs after, $\left.F_{(1,38)}=0.01, \mathrm{NS}\right)$, nor a significant interaction between group or time $\left(F_{(1,38)}=0.43\right.$, NS).

Rather, the change in slow-wave-associated activity in the odor-exposed group was linked to a shift in temporal structure of single-unit activity after odor exposure. To analyze this, we focused on the histogram bin showing the maximal (peak) activity for each unit during the two slow-wave sampling periods. An example of a single-unit shifting its slow-wave-associated activity is shown in Figure 2D. Although single units in both conditions showed some variability in the timing of peak activity during slow waves, single units in odor-exposed animals showed a signifi- subtracted from mean pre-waveform within each animal) showed a significant increase in variability of this measure in the odor-exposed group (ANOVA, group $\times$ time interaction, $F_{(9,117)}$ $=65.66, p<0.001)$. Specifically, despite the lack of change in mean morphology of slow-waves before versus after odor, variability in the early phase of the waveform was significantly enhanced by odor experience. Variability in the control waveforms was constant across the waveform and significantly less than that observed in the early phase of the slow waves in odor-exposed animals. As with the PSWTH analyses, this enhancement in variation may reflect the fact that some waveforms increased and some decreased in early phases after odor exposure.

\section{Discussion}

Activity within the aPCX during slow-wave states is shaped by recent odor experience. The timing of single-unit activity relative to the population of cells active during a slow wave was modified if the animal had recently (past several minutes) been stimulated with an odor within the receptive field of that cell. If no odor had been delivered or the cell did not respond to presented odors, the activity of the cell during SWA was stable. Furthermore, the slow wave itself became more variable, especially along its leading edge, after odor stimulation. No such change was observed in control animals. These results are consistent with memory for recently experienced odors being expressed during spontaneous SWA within the aPCX. Whether this experience-dependent SWA represents a replay or reactivation (Skaggs and McNaughton, 
1996; Lee and Wilson, 2002) of a learned odor object or whether SWA is involved in consolidation of olfactory perceptual learning that can occur using similar stimulation protocols in anesthetized rats (Wilson, 2003) is currently being examined. These results suggest, however, a potential similarity between the role of slowwave states in the aPCX and hippocampal formation.

During SWA, aPCX single-unit activity occurred primarily in-phase with LFP slow waves. Individual cells varied in the specific temporal structure of their firing activity relative to the slow waves under baseline conditions, and, in control cells, this temporal structure was relatively stable over many minutes and between consecutive slow-wave periods, as measured by overall PSWTH and PSWTH peaks. However, if cells had been activated by odor stimulation in FWA, those cells shifted their activity relative to the population (LFP slow wave) during the subsequent slow-wave period, just as might be expected if the activity of those cells was modified by the previous odor exposure.

Previous work has demonstrated that aPCX acts much like thalamus during SWA to serve as a state-dependent sensory gate (Murakami et al., 2005). During SWA, despite relatively maintained input from mitral/tufted cells of the olfactory bulb, piriform cortical neurons show reduced responsivity to afferent input (Murakami et al., 2005) (Fig. 1). This reduced cortical activity may underlie the observed decrease in perceptual responses to odors during slow-wave sleep (Carskadon and Herz, 2004). The present results suggest that, similar to thalamocortical and hippocampal systems, during this period of reduced sensitivity to afferent input, the aPCX turns inward, with activity driven by intracortical associative synapses. A change in the effectiveness of intrinsic synapses during SWA could occur via changes in cholinergic modulation (Hasselmo and Bower, 1992; Liljenström and Hasselmo, 1995; Hasselmo, 1999). Acetylcholine suppresses intrinsic association fiber synapses in aPCX (Hasselmo and Bower, 1992), effectively enhancing the relative importance of afferent input on cortical activity during periods of high cholinergic activity (FWA). Critically, cholinergic input to many forebrain regions drops during SWA, allowing a shift toward intrinsic activity dominance (Liljenström and Hasselmo, 1995; Hasselmo, 1999). The most effective synapses during this time may be those that have been strengthened by recent experience. Reactivation of these recently strengthened synapses during slow-wave states may help consolidate the newly initiated changes and, in so doing, help consolidate memory critical for enhanced acuity or odor associations.

The present results have at least two important implications. First, they suggest that the role of slow-wave states in memory observed in hippocampal circuits may be a common feature of other similar circuits such as aPCX. Second, the olfactory system offers some unique advantages for the study of SWA and memory. Odors, which are complex stimulus patterns (Lin et al., 2006), can be quantitatively manipulated, delivered to animals in different behavioral states, and be rapidly learned and remembered for long times. In fact, odor memory can even be induced rapidly under anesthesia (Wilson, 2003; Wilson et al., 2004). Thus, the olfactory system may be an especially powerful new model system for the study of slow-wave sleep and memory consolidation.

\section{References}

Barkai E, Hasselmo ME (1994) Modulation of the input/output function of rat piriform cortex pyramidal cells. J Neurophysiol 72:644-658.

Barkai E, Bergman RE, Horwitz G, Hasselmo ME (1994) Modulation of associative memory function in a biophysical simulation of rat piriform cortex. J Neurophysiol 72:659-677.
Buzsáki G (1996) The hippocampo-neocortical dialogue. Cereb Cortex 6:81-92.

Cai DJ, Shuman T, Gorman MR, Sage JR, Anagnostaras SG (2009) Sleep selectively enhances hippocampus-dependent memory in mice. Behav Neurosci 123:713-719.

Carskadon MA, Herz RS (2004) Minimal olfactory perception during sleep: why odor alarms will not work for humans. Sleep 27:402-405.

Chaudhury D, Escanilla O, Linster C (2009) Bulbar acetylcholine enhances neural and perceptual odor discrimination. J Neurosci 29:52-60.

Cohen Y, Reuveni I, Barkai E, Maroun M (2008) Olfactory learninginduced long-lasting enhancement of descending and ascending synaptic transmission to the piriform cortex. J Neurosci 28:6664-6669.

Doucette W, Restrepo D (2008) Profound context-dependent plasticity of mitral cell responses in olfactory bulb. PLoS Biol 6:e258.

Doucette W, Milder J, Restrepo D (2007) Adrenergic modulation of olfactory bulb circuitry affects odor discrimination. Learn Mem 14:539-547.

Eschenko O, Ramadan W, Mölle M, Born J, Sara SJ (2008) Sustained increase in hippocampal sharp-wave ripple activity during slow-wave sleep after learning. Learn Mem 15:222-228.

Fletcher ML, Wilson DA (2002) Experience modifies olfactory acuity: acetylcholine-dependent learning decreases behavioral generalization between similar odorants. J Neurosci 22:RC201(1-5).

Fletcher ML, Wilson DA (2003) Olfactory bulb mitral-tufted cell plasticity: odorant-specific tuning reflects previous odorant exposure. J Neurosci 23:6946-6955

Grajski KA, Freeman WJ (1989) Spatial EEG correlates of nonassociative and associative olfactory learning in rabbits. Behav Neurosci 103:790-804.

Haberly LB (2001) Parallel-distributed processing in olfactory cortex: new insights from morphological and physiological analysis of neuronal circuitry. Chem Senses 26:551-576.

Hasselmo ME (1999) Neuromodulation: acetylcholine and memory consolidation. Trends Cogn Sci 3:351-359.

Hasselmo ME, Bower JM (1992) Cholinergic suppression specific to intrinsic not afferent fiber synapses in rat piriform (olfactory) cortex. J Neurophysiol 67:1222-1229.

Johnson BA, Leon M (2007) Chemotopic odorant coding in a mammalian olfactory system. J Comp Neurol 503:1-34.

Kadohisa M, Wilson DA (2006) Separate encoding of identity and similarity of complex familiar odors in piriform cortex. Proc Natl Acad Sci U S A 103:15206-15211.

Lee AK, Wilson MA (2002) Memory of sequential experience in the hippocampus during slow wave sleep. Neuron 36:1183-1194.

Li W, Howard JD, Parrish TB, Gottfried JA (2008) Aversive learning enhances perceptual and cortical discrimination of indiscriminable odor cues. Science 319:1842-1845.

Liljenström H, Hasselmo ME (1995) Cholinergic modulation of cortical oscillatory dynamics. J Neurophysiol 74:288-297.

Lin da Y, Shea SD, Katz LC (2006) Representation of natural stimuli in the rodent main olfactory bulb. Neuron 50:937-949.

Linster C, Menon AV, Singh CY, Wilson DA (2009) Odor-specific habituation arises from interaction of afferent synaptic adaptation and intrinsic synaptic potentiation in olfactory cortex. Learn Mem 16:452-459.

Louie K, Wilson MA (2001) Temporally structured replay of awake hippocampal ensemble activity during rapid eye movement sleep. Neuron 29:145-156.

Mandairon N, Stack C, Kiselycznyk C, Linster C (2006) Broad activation of the olfactory bulb produces long-lasting changes in odor perception. Proc Natl Acad Sci U S A 103:13543-13548.

Maquet P (2001) The role of sleep in learning and memory. Science 294:1048-1052.

Martin C, Gervais R, Messaoudi B, Ravel N (2006) Learning-induced oscillatory activities correlated to odour recognition: a network activity. Eur J Neurosci 23:1801-1810.

Martin C, Beshel J, Kay LM (2007) An olfacto-hippocampal network is dynamically involved in odor-discrimination learning. J Neurophysiol 98:2196-2205.

Murakami M, Kashiwadani H, Kirino Y, Mori K (2005) State-dependent sensory gating in olfactory cortex. Neuron 46:285-296.

Patil MM, Linster C, Lubenov E, Hasselmo ME (1998) Cholinergic agonist carbachol enables associative long-term potentiation in piriform cortex slices. J Neurophysiol 80:2467-2474. 
Pavlides C, Winson J (1989) Influences of hippocampal place cell firing in the awake state on the activity of these cells during subsequent sleep episodes. J Neurosci 9:2907-2918.

Poo C, Isaacson JS (2009) Odor representations in olfactory cortex: "sparse" coding, global inhibition, and oscillations. Neuron 62:850-861.

Quinlan EM, Lebel D, Brosh I, Barkai E (2004) A molecular mechanism for stabilization of learning-induced synaptic modifications. Neuron 41:185-192.

Ravel N, Chabaud P, Martin C, Gaveau V, Hugues E, Tallon-Baudry C, Bertrand O, Gervais R (2003) Olfactory learning modifies the expression of odour-induced oscillatory responses in the gamma $(60-90 \mathrm{~Hz})$ and beta $(15-40 \mathrm{~Hz})$ bands in the rat olfactory bulb. Eur J Neurosci 17:350-358.

Rennaker RL, Chen CF, Ruyle AM, Sloan AM, Wilson DA (2007) Spatial and temporal distribution of odorant-evoked activity in the piriform cortex. J Neurosci 27:1534-1542.

Skaggs WE, McNaughton BL (1996) Replay of neuronal firing sequences in rat hippocampus during sleep following spatial experience. Science 271:1870-1873.

Steriade M, Timofeev I (2003) Neuronal plasticity in thalamocortical networks during sleep and waking oscillations. Neuron 37:563-576.

Stevenson RJ (2001) Perceptual learning with odors: implications for psychological accounts of odor quality perception. Psychon Bull Rev $8: 708-712$.
Wachowiak M, Cohen LB (2001) Representation of odorants by receptor neuron input to the mouse olfactory bulb. Neuron 32:723-735.

Wierzynski CM, Lubenov EV, Gu M, Siapas AG (2009) State-dependent spike-timing relationships between hippocampal and prefrontal circuits during sleep. Neuron 61:587-596.

Wilson DA (1998) Habituation of odor responses in the rat anterior piriform cortex. J Neurophysiol 79:1425-1440.

Wilson DA (2000) Comparison of odor receptive field plasticity in the rat olfactory bulb and anterior piriform cortex. J Neurophysiol 84:3036-3042.

Wilson DA (2001) Scopolamine enhances generalization between odor representations in rat olfactory cortex. Learn Mem 8:279-285.

Wilson DA (2003) Rapid, experience-induced enhancement in odorant discrimination by anterior piriform cortex neurons. J Neurophysiol 90:65-72.

Wilson DA, Stevenson RJ (2003) Olfactory perceptual learning: the critical role of memory in odor discrimination. Neurosci Biobehav Rev 27: 307-328.

Wilson DA, Sullivan RM, Leon M (1987) Single-unit analysis of postnatal olfactory learning: modified olfactory bulb output response patterns to learned attractive odors. J Neurosci 7:3154-3162.

Wilson DA, Best AR, Sullivan RM (2004) Plasticity in the olfactory system: lessons for the neurobiology of memory. Neuroscientist 10:513-524. 